\title{
Muscle strength field-based tests to identify European adolescents at risk of metabolic syndrome: The HELENA study
}

José Castro-Piñero $^{\mathrm{a}^{*}}$, Kelly R. Laurson ${ }^{\mathrm{b}}$, Enrique G. Artero ${ }^{\mathrm{c}}$, Francisco B Ortega ${ }^{\mathrm{d}, \mathrm{e}}$, Idoia Labayen ${ }^{\mathrm{f}}$, Azahara I. Ruperez ${ }^{\mathrm{g}}$, Mahmoud Zaqout ${ }^{\mathrm{h}}$, Yannis Manios ${ }^{\mathrm{i}}$, Jeremy Vanhelst ${ }^{\mathrm{j}}$, Ascension Marcos $^{\mathrm{k}}$, Angela Polito ${ }^{1}$, Marcela Gonzalez-Gross ${ }^{\mathrm{m}}$, Kurt Widhalm $^{\mathrm{n}}$, Luis A Morenog, Angel Gutierrez ${ }^{\mathrm{o}}$, Jonatan R Ruiz ${ }^{\mathrm{d}, \mathrm{e}}$.

\section{Affiliation:}

aDepartment of Physical Education, Faculty of Education Sciences, University of Cádiz, Puerto Real, Spain.

${ }^{\mathrm{b}}$ School of Kinesiology and Recreation, Illinois State University.

c SPORT Research Group (CTS-1024), University of Almería, Almería, Spain.

d PROmoting FITness and Health through Physical Activity Research Group (PROFITH), Department of Physical Education and Sports, Faculty of Sport Sciences, University of Granada, Granada, Spain.

e Department of Biosciences and Nutrition at NOVUM, Karolinska Institutet, Huddinge, Sweden.

${ }^{\mathrm{f}}$ Department of Health Sciences, Public University of Navarra, Pamplona, Spain.

g GENUD (Growth, Exercise, NUtrition and Development) Research Group, Facultad de Ciencias de la Salud, Instituto Agroalimentario de Aragón (IA2), Universidad de Zaragoza, Instituto de Investigación Sanitaria de Aragón (IIS Aragón) and Centro de Investigación Biomédica en Red de Fisiopatología de la Obesidad y Nutrición (CIBERObn), Spain. 
h Department of Public Health, Faculty of Medicine and Health Sciences, Ghent University, Ghent, Belgium.

${ }^{\text {i }}$ Department of Nutrition and Dietetics, Harokopio University, Athens, Greece.

¡ Univ. Lille, Inserm, CHU Lille, U995 - LIRIC - Lille Inflammation Research

International Center, CIC 1403 - Centre d'investigation clinique, F-59000, Lille, France.

${ }^{\mathrm{k}}$ Immunonutrition Research Group, Department of Metabolism and Nutrition, Instituto del Frio, Institute of Food Science, Technology and Nutrition, Spanish National Research Council, Madrid Spain.

${ }^{1}$ National Institute for Food and Nutrition Research, Rome, Italy.

${ }^{\mathrm{m}}$ ImFine Research Group. Facultad de Ciencias de la Actividad Física y del DeporteINEF, Universidad Politécnica de Madrid, Madrid, Spain, and CIBER: CB12/03/30038 Fisiopatología de la Obesidad y la Nutrición, CIBERobn, Instituto de Salud Carlos III (ISCIII), Spain.

n Department of Pediatrics, Division of Clinical Nutrition, Medical University of Vienna, Vienna, Austria.

${ }^{\circ}$ Department of Medical Physiology, School of Medicine, University of Granada, Spain

\section{Corresponding author:}

José Castro-Piñero, Department of Physical Education, School of Education, University of Cádiz, 11519, Puerto Real, Spain, jose.castro@uca.es, telephone: +34 607704404.

Word count: 3578

Abstract word count: 233

Number of Table: 3 


\section{ABSTRACT}

2 Objectives: To determine whether handgrip strength (HG) and/or standing long jump (SLJ) are

3 capable of detecting risk of metabolic syndrome (MetS) in European adolescents, and to identify

4 age- and sex-specific cut points for these tests.

5 Design: Cross-sectional study.

6 Methods: Participants included 969 (aged 12.5-17.5 years old) adolescents from 9 European

7 countries ( $n=520$ girls). Absolute and relative HG and SLJ tests were used to assess upper and

8 lower muscle strength, respectively. MetS status was determined using the age- and sex-specific

9 cut points proposed by Jolliffe and Janssen's, Additionally, we computed a continuous

10 cardiometabolic risk index with the average z-score of four cardiometabolic risk factors: Wait

11 circumference, mean arterial pressure, triglycerides/high-density lipoprotein cholesterol, and

12 fasting insulin.

13 Results: The prevalence of MetS was 3.1\% in European adolescents. Relative HG and absolute

14 SLJ were the best tests for detecting the presence of MetS (Area under the receiver operating 15 characteristic $(A U C)=0.799, \quad 95 \% C I: 0.773-0.824 ;$ and $A U C=0.695 \quad 95 \% C I: 0.665-0.724)$,

16 respectively) and elevated cardiometabolic risk index ( $A U C=0.873,95 \% C I: 0.838-0.902$; and

$17 A U C=0.72895 \% C I: 0.698-0.756)$, respectively) and, regardless of cardiorespiratory fitness. We

18 provide age- and sex-specific cut points of upper and lower muscle strength for European

19 adolescents to identify the presence of MetS and elevated cardiometabolic risk index.

20 Conclusions: The proposed health-related cut points could be used as a starting point to define

21 health-related levels of upper and lower muscle strength in adolescents. Likewise, the diagnostic

22 statistics provided herein can be used to offer feedback to adolescents, parents, and education

23 and health professionals about what it means to meet or fail test standards. 
24 Key words: Muscle fitness. Cardiometabolic risk. Adolescents

25

26

27

28

29

30

31

32

33

34

35

36

37

38

39

40

41

42

43

44

45

46 


\section{Introduction}

48 Metabolic syndrome (MetS) has become a major health challenge worldwide with its prevalence

49 increasing in concert with obesity and sedentary lifestyles. ${ }^{1}$ MetS is defined as clustering of

50 dichotomous or continuous cardiometabolic risk factors, which includes dyslipidemia

51 (triglycerides and cholesterol), hypertension, glucose intolerance, and total and/or central

52 adiposity. ${ }^{1}$ MetS affects both youth and adults and has been associated with cardiovascular

53 disease and type 2 diabetes, ${ }^{2}$ as well as with all-cause mortality in non-diabetic individuals and in

54 adult populations. ${ }^{1}$ Given that MetS and many of its features track from childhood into

55 adulthood, ${ }^{2}$ early detection and diagnosis of MetS in youth is necessary to develop effective

56 prevention programs.

57 Both upper and lower body muscle strength levels are considered important markers of 58 cardiometabolic health in children and adolescents. ${ }^{3}$ Moreover, muscle strength is associated

59 with cardiometabolic risk factors, independently of cardiorespiratory fitness. ${ }^{4}$ The Institute Of

60 Medicine $^{5}$ recommended that a survey of health-related physical fitness in youth should include

61 upper and lower body muscle strength measurements. Furthermore, this Institute called for the

62 need of determining health-related muscle strength cut points for children and adolescents for

63 identifying youth who may benefit from primary and secondary cardiometabolic prevention

64 programming. 5

65 The Healthy Lifestyle in Europe by Nutrition in Adolescence (HELENA) study is a multicenter,

66 cross-sectional study performed in nine European countries primarily designed to obtain reliable

67 and comparable data on nutrition and health-related parameters of a relatively large sample of

68 European adolescents aged 12.5-17.5..$^{6}$ The HELENA study collected data on upper and lower

69 body muscle strength measured by means of the handgrip strength (HG) and the standing long 
70 jump (SLJ) tests. Both tests have been proposed to assess upper and lower body muscle strength 71 levels in European youth. ${ }^{7}$ In addition, the HELENA study also collected data on MetS and other 72 relevant clinical and socio-demographic features in a large sample of European adolescents, thus

73 providing a great opportunity (i) to determine whether the HG and/or SLJ tests are capable of 74 detecting risk of MetS in European adolescents, and (ii) to identify age- and sex-specific cut 75 points for these tests.

76

77

78

79

80

81

82

83

84

85

86

87 


\section{Methods}

Adolescents volunteered to participate in the HELENA study, a multicenter cross-

90 sectional study on lifestyle and nutrition, conducted in 10 European cities (cluster) from 9

91 European countries (Vienna, Ghent, Lille, Dortmund, Athens, Heraklion, Pécs, Rome, Zaragoza

92 and Stockholm). ${ }^{6}$

93 The HELENA study sample comprised 3,528 adolescents (52\% girls) aged $12.5-17.5$

94 years old. Blood sampling was randomly performed in one-third of the recruited adolescents

$95(n=1,089)$. The present study included adolescents who had complete data on body mass index

96 (BMI), muscle strength, cardiorespiratory fitness, and the cardiometabolic risk factors considered

97 in these analyses: waist circumference (WC), diastolic and systolic blood pressure, triglycerides

98 (TG), high-density lipoprotein cholesterol (HDL), and fasting glucose and insulin levels. The

99 sample sizes vary by analysis (see all tables), but 1,574 and 1567 boys contributed physical

100 fitness data on HG and SLJ, respectively, and 1,718 and 1702 girls contributed physical fitness

101 data on HG and SLJ, respectively, whereas 449 boys and 520 girls also had blood data for the

102 analyses. $^{8}$

103 The study was approved by the Research Ethics Committees of each study site, and was

104 performed following the ethical guidelines of the Declaration of Helsinki 1964 (revision of

105 Edinburgh 2000). A written informed consent was obtained from the parents of the adolescents

106 and the adolescents themselves.

107 Body mass index

108 Body mass was measured in underwear without shoes using an electronic scale (Type

109 SECA 861, Hamburg, Germany) to the nearest $0.1 \mathrm{~kg}$. Stature was measured barefoot to the

110 nearest $0.1 \mathrm{~cm}$ using the Frankfort horizontal plane and a stadiometer (Type SECA 225, 
111 Hamburg, Germany). BMI was calculated as body mass $(\mathrm{kg}) /$ stature $(\mathrm{m})^{2}$. The International

112 Obesity Task Force BMI standards were used to categorize children as normal weight or

113 overweight/obese. ${ }^{9}$

114 Physical Fitness

115 Upper and lower body muscle strength levels were measured by the HG and the SLJ tests, ${ }^{7}$ 116 respectively. Both test are valid ${ }^{10}$, reliable ${ }^{11}$, feasible and safe ${ }^{12}$ to be used both at population

117 level and in the school-setting. ${ }^{7}$ A hand dynamometer with an adjustable grip (TKK 5101 Grip

118 D, Takey, Tokyo, Japan) was used for the HG test. The adolescent squeezed the dynamometer

119 continuously for at least 2 seconds, alternatively with right and left hands, with the elbow in full

120 extension. The grip-span of the dynamometer was adjusted according to the hand size of the

121 adolescent. The test was performed twice, allowing a 1-minute rest between the measurements to

122 avoid local muscle fatigue, and the maximum score for each hand was recorded in kilograms as

123 described elsewhere. ${ }^{13}$ The average of the scores achieved by the left and right hands was used in

124 the analyses to have an overall measure of the handgrip strength. ${ }^{13}$ The SLJ was performed from

125 a starting position immediately behind a line, standing with feet approximately shoulder's width

126 apart, and the adolescent jumped as far forward as possible, landing with their feet together. The

127 test was performed twice, with 1-minute rest between the measurements, and the longest distance

128 achieved was recorded in centimeters. Before conducting the tests, adolescents had a

129 familiarization trial. Nevertheless, as these tests are commonly used in the school setting to

130 measure fitness performance, adolescents were rather familiarized. We converted HG and SLJ to

131 relative scores by expressing HG as strength divided by body mass [strength $(\mathrm{kg})$ / body mass

132 (kg)] and expressing SLJ as jump distance multiplied by body mass [jump distance (cm) $\mathrm{x}$ body 133 mass $(\mathrm{kg})]$. 
134 In order to determine the potential influence of cardiorespiratory fitness (a well-known important 135 marker of health in adolescents ${ }^{3}$ on the association of HG and/or SLJ with the risk of MetS, we

136 decided to control the analysis by this variable. We assessed cardiorespiratory fitness by the 20

$137 \mathrm{~m}$ shuttle run test and the maximum oxygen consumption $\left(\mathrm{VO}_{2 \mathrm{max}}, \mathrm{ml} / \mathrm{kg} / \mathrm{min}\right)$ by the equation

138 reported by Léger et al. ${ }^{14}$ Each adolescent was grouped into a cardiorespiratory fitness status

139 (low or high) according to the FITNESSGRAM standards Healthy Fitness Zone as follows: low

140 corresponds to the "needs improvement" category, and high correspond to the "healthy fitness

141 zone". ${ }^{15}$ All these fitness tests have shown to be valid and reliable in children and adolescents. ${ }^{10}$,

$142 \quad 11$

143 Cardiometabolic risk factors

144 Waist circumference was measured in triplicate using an anthropometric tape (SECA 200) as the 145 midpoint between the lowest rib and the iliac crest. ${ }^{16}$ Diastolic and systolic blood pressure were 146 measured after being seated in a quiet room for 10 min with their back supported and feet on the

147 ground. Two diastolic and systolic blood pressure readings were taken with a 10-min interval of 148 quiet rest. The lower value of the two measurements was used in the analysis. We calculated the 149 mean arterial pressure as [diastolic blood pressure $+(0.333 \mathrm{x}$ (systolic blood pressure - diastolic 150 blood pressure))]. A detailed description of the blood samples' analysis has been reported 151 elsewhere. ${ }^{17}$ Venous blood was obtained by venipuncture after an overnight fast. Serum TG, 152 HDL, and fasting glucose were measured on a Dimension RxL clinical chemistry system (Dade 153 Behring, Schwalbach, Germany) using enzymatic methods. Fasting insulin concentrations were 154 measured by a solid-phase two-site chemiluminescent immunometric assay, using an Immulite 1552000 analyzer (DPC Biermann GmbH, Bad Nauheim, Germany). 
MetS status was also determined using WC, diastolic and systolic blood pressure, TG,

157 HDL, and fasting glucose with the definition by Jolliffe and Janssen reported in 2007. ${ }^{18}$ This

158 pediatric definition was created using growth curves to back-extrapolate the National Cholesterol

159 Education Program/Adult Treatment Panel II adult values for adolescents. The participants were

160 considered as having an individual elevated cardiometabolic risk factor if they had a high WC,

161 either systolic or diastolic blood pressure, TG, HDL, or fasting glucose. Adolescents with 3 or 162 more elevated cardiometabolic risk factors were considered as having MetS.

163 Additionally, all cardiometabolic risk factors were expressed as age- and sex-specific z-

164 scores based on the current sample to account for changes during growth and maturation.

165 Further, a continuous cardiometabolic risk index was computed as the average z-score of four

166 cardiometabolic risk factors (WC, mean arterial pressure, TG/HDL ratio, and fasting insulin).

167 The adolescents were categorized as having elevated cardiometabolic risk if their

168 cardiometabolic risk index was one standard deviation above the mean for each of the four

169 markers. This cardiometabolic risk index methodology has been previously validated in children

170 and adolescents. ${ }^{19}$

171 The descriptive data are shown as mean and standard deviation unless otherwise

172 indicated, and the sexes were compared with independent samples t-tests and chi-square tests of

173 independence. Receiver operating characteristic (ROC) analyses were completed for all four

174 muscle strength parameters: absolute HG, relative HG (HG / body mass), absolute SLJ, and

175 relative SLJ (SLJ x body mass). The Least Mean Square (LMS) method ${ }^{20}$ was used to create age-

176 and sex-specific z-scores and were used in the main analysis.

In the current study, we constructed ROC curves to detect MetS and elevated

178 cardiometabolic risk index from the four muscle strength parameters. The resulting ROC curves 
and data provide several key variables that aid in identifying appropriate thresholds, such as: area under the ROC curve $(A U C)$, sensitivity, specificity, Youden Index (i.e. the sum of sensitivity and specificity minus one, and is the most commonly used indicator of an ideal cut point on the ROC curve), positive predictive value ( $P P V)$, negative predictive value $(N P V)$, and the diagnostic odds ratio $(O R)^{21}$ In terms of this analysis, $A U C$ is a test of global discriminatory accuracy indicating how well the muscle strength z-score can differentiate between MetS vs. noMetS and elevated vs. low cardiometabolic risk index. sample to identify the impact of combining both sexes. After creating the curves, the four tests of muscle strength were compared to identify the best tests/metrics to use for the intended purpose: whether absolute or relative HG, and absolute or relative SLJ. Pairwise comparisons of the AUC 190 values were made using the methods outlined by Hanley and McNeil. ${ }^{22}$ Then, to select the ideal 191 cut points for each of the four tests, we primarily made decisions based on the Youden Index, but 192 we also gave consideration to the $P P V, N P V$, and diagnostic $O R$ for each threshold. After 193 selecting the ideal cut points, to determine how the predictive utility of the thresholds would be 194 impacted by cardiorespiratory fitness, we used logistic regression to estimate the odds of MetS 195 and elevated cardiometabolic risk index in youth with muscle strength cut points (low vs. high 196 levels) in two models: unadjusted or adjusted for cardiorespiratory fitness status. The LMS 197 percentile curves and ROC curves were constructed using LMS ChartMaker Pro (version 2.3). 198 All other analyses were done using IBM SPSS (version 20.0). The alpha level for all analyses 199 was set at $p \leq 0.05$. 


\section{Results}

Boys had higher levels of absolute and relative upper and lower body muscle strength

204 than girls (all $p<0.05$ ), as well as higher WC, systolic blood pressure, mean arterial pressure, 205 and fasting glucose (all $p<0.05$ ). However, girls had higher levels of TG and HDL than boys 206 (both $p<0.05$ ). The prevalence of MetS as well as the elevated cardiometabolic risk index was 207 similar in boys and girls (Table S1).

Table S2 shows the $A U C$ and pairwise comparisons of the four muscle strength 209 parameters calculated. All AUCs, except for absolute HG to predict MetS in girls, were 210 significantly different from a non-informative test (all $p<0.05$ ), indicating that any of the four 211 parameters could be used to differentiate between those with MetS and with elevated 212 cardiometabolic risk index. The $A U C$ values were higher for boys than for girls, and the $A U C$ for 213 specific curves ranged from moderately accurate ( 0.873 for boys' relative $\mathrm{HG}$ ) to less accurate 214 (0.539 for girls' absolute HG). For both boys and girls, the relative HG was a significantly better 215 indicator of MetS, and the elevated cardiometabolic risk index (i.e., $\geq 1$ z-score) than absolute 216 HG. Furthermore, there was no difference in the $A U C$ between absolute and relative SLJ. In 217 general, the more informative iterations of the muscle strength parameters were relative HG and 218 absolute SLJ.

219 The selected thresholds for each muscle strength test to identify an elevated 220 cardiometabolic risk index are shown in Table 1. Each cut point selected as 'ideal' had the 221 highest Youden Index of the potential thresholds, except girls' absolute SLJ. For girls' absolute 222 SLJ, the highest Youden Index was found at a -0.0183 z-score (approximately the $49^{\text {th }}$ 223 percentile). However, the $5^{\text {th }}$ highest Youden Index $(\mathrm{z}$-score $=-0.846)$ had a higher specificity, 
$224 P P V$, and diagnostic $O R$. Because it afforded these advantages and was still within the top $1 \%$ of 225 Youden Index scores it was selected instead.

226 Table 2 depicts the selected thresholds for each muscle strength test to identify MetS.

227 Each 'ideal' cut point had the highest Youden Index available except two boys' thresholds, 228 relative HG and absolute SLJ. For both, the cut point with the highest Youden Index was 229 relatively unbalanced, a very low specificity for relative HG (z-score $=-0.4847)$ and a high 230 specificity for absolute SLJ (z-score $=-1.5557)$. The relative HG and absolute SLJ cut points 231 selected for boys where those with the higher sensitivities, specificities, $P P V, N P V$, and odds 232 ratios (z-score $=-1.127$ and -0.890 , respectively). Moreover, Youden Index scores were near the 233 top of the possible cut points. The diagnostic ORs were higher for boys than girls. It should be 234 noted that the cut points for absolute HG and relative SLJ are reversed from what would be 235 considered intuitive. Higher absolute HG and relative SLJ scores were more indicative of greater 236 odds of having MetS or an elevated cardiometabolic risk index.

237 Table 3 outlines the selected age- and sex-specific scores for relative HG and absolute 238 SLJ for boys and girls derived in the current study. These approximate the $25^{\text {th }}$ and $20^{\text {th }}$ 239 percentiles using the LMS parameters for relative HG and absolute SLJ, respectively. These final 240 cut points are based on the 'ideal' cut points for boys and girls from Tables 2 and 3, where the 241 relative HG cut points for boys and girls were the $25.1^{\text {th }}$ and $26.7^{\text {th }}$ percentiles, and the absolute 242 SLJ cut points were the $21.5^{\text {th }}$ and $21.3^{\text {rd }}$.

244 cardiometabolic risk index (OR: 6.2, 95\% CI: 2.9-13.4; and $O R:$ 8.5, 95\% CI: 5.0-14.7, 245 respectively) than those with scores at or above the determined cut points. Likewise, boys and 246 girls with low absolute SLJ scores were more likely to have MetS and elevated cardiometabolic 
247 risk index (OR: 4.5, 95\% CI: 2.3-9.4; and $O R: 5.8,95 \%$ CI: 3.5-9.6; respectively) than those with 248 scores at or above the determined cut points. However, these $O R$ s were attenuated, but were still 249 statistically significant when adjusting for cardiorespiratory fitness status, for both relative HG 250 (OR: 5.2, 95\% CI: 2.4-11.5 for MetS; and OR: 7.3, 95\% CI: 4.2-12.7 for cardiometabolic risk 251 index) and absolute SLJ (OR: 3.6 95\% CI: 1.7-7.7 for MetS; and OR: 4.7, 95\% CI: 2.8-7.9 for 252 cardiometabolic risk index).

253

254

255

256

257

258

259

260

261

262

263

264

265

266 


\section{Discussion}

The aim of the present study was to determine whether HG and/or SLJ are capable of detecting risk of MetS in European adolescents, and to identify age- and sex-specific cut points

270 for these tests. The main findings were that (1) the prevalence of MetS and elevated 271 cardiometabolic risk index was 3.1 and 7.2\% in European adolescents from 9 countries,

272 respectively; (2) relative $\mathrm{HG}$ and absolute SLJ were the best muscle strength tests for detecting

273 MetS and elevated cardiometabolic risk; (3) the identified muscle strength for detecting MetS

274 and elevated cardiometabolic risk index were identical, which further reinforce the existence of a

275 muscle threshold associated with cardiovascular health in youth; (4) age- and sex-specific health-

276 related cut points were provided for European adolescents in order identify MetS and elevated

277 cardiometabolic risk, which seems to be more discriminative for boys than for girls. This is the

278 first study that establishes age- and sex-related health-related upper and lower muscle strength in

279 adolescents.

280 The prevalence of MetS in children and adolescents varied between $0 \%$ and $60 \%$, 281 depending on the definition of MetS and the population examined. ${ }^{23}$ For the pediatric population, 282 there is a lack of a uniform definition. Many different MetS criteria have been applied in 283 adolescents, and the components and cut points used to diagnose the MetS have varied 284 considerably among studies. ${ }^{18,24}$ Several studies have used modified criteria based on the same 285 concept in adults, according to Program/Adult Treatment Panel III $^{25}$ and the International 286 Diabetes Federation. ${ }^{1}$ These definitions are based on dichotomization of the cardiometabolic risk 287 factors, and to be clinically diagnosed with MetS at least three cardiometabolic risk factors must 288 be achieved, including obesity. However, other studies have established clustering of 289 cardiometabolic risk factors, using continuous scores. Recently, Andersen et al. ${ }^{24}$ showed that 
290 more children and adolescents had clustering of cardiometabolic risk factors $(6.2 \%$ had 4 or 291 more cardiometabolic risk factors) than the number fulfilling the International Diabetes 292 Federation definition of MetS (less than 1\%) for children and adolescents. In the present study, 293 we included both methods, MetS and cardiometabolic risk index. For the dichotomous method, 294 we used the model developed by Joliffe and Janssen, ${ }^{18}$ who created age-specific cut points and 295 MetS criteria for adolescents that were linked to the health-based Program/Adult Treatment 296 Panel III and International Diabetes Federation adult criteria. For the continuous method, we 297 chose the valid model by Martínez-Vizcaino et al. ${ }^{19}$ who used confirmatory factor analysis 298 comparing with other continuous methods. We observed that 3.1\% had MetS and 7.2\% European 299 adolescents had elevated cardiometabolic risk index, which concurs with the figures reported by $300 \quad$ Andersen et al. ${ }^{24}$

301 The present study examined whether either HG or SLJ tests were capable of detecting 302 elevated MetS and elevated cardiometabolic risk index in European adolescents. We selected the 303 relative HG and absolute SLJ because the $A U C$ value was higher for relative HG than for 304 absolute HG. Moreover, although there was no difference in the $A U C$ values between absolute 305 and relative SLJ, the other discriminatory parameters showed that absolute SLJ identified 306 thresholds to diagnose MetS and elevated cardiometabolic risk more accurately. Moreover, we 307 excluded absolute HG and relative SLJ thresholds from any further analyses because we found 308 positive associations with MetS and elevated cardiometabolic risk. If implemented (as part of a 309 fitness testing program), adolescents with higher absolute HG or higher relative SLJ scores 310 would actually be grouped in the 'unhealthy zones'. It must be noted that heavier individuals 311 have higher levels of absolute HG and relative SLJ, and the prevalence of MetS is higher in 312 obese as opposed to normal weight children and adolescents, increasing with severity of 
313 obesity. ${ }^{26}$ In addition, regarding the validity of the muscle strength tests, it is assumed that only

314 non-weight-bearing fitness tests should be normalized by body mas. ${ }^{27}$

315 We reported age- and sex-specific relative HG and absolute SLJ cut points selected as the

316 most accurate to detect MetS, and elevated cardiometabolic risk index in a relatively large

317 sample of European adolescents. Ramirez-Velez et al. ${ }^{28}$ developed age group-sex-specific cut

318 points of relative HG for optimal cardiometabolic risk categorization in children (9-12.9 years

319 old) and adolescents (13-17.9 years old) from Bogota (Colombia). In adolescent boys, the cut

320 point reported by Ramirez-Velez et al. ( $0.447 \mathrm{~kg} /$ body mass) was similar to the one we

321 established for 13-year-old adolescent boys. However, in girls, the cut point reported by

322 Ramirez-Velez et al. (0.440 kg/body mass) was slightly higher than the ones we calculated for

323 our European girls. Moreover, Peterson et al. ${ }^{29}$ reported a high-risk cardiometabolic threshold for

324 boys ( $\leq 0.33 \mathrm{~kg} /$ body mass) and girls ( $\leq 0.28 \mathrm{~kg} /$ body mass), an intermediate threshold (boys, $>$

3250.33 and $\leq 0.45 \mathrm{~kg} /$ body mass; girls, $>0.28$ and $\leq 0.36 \mathrm{~kg} /$ body mass), as well as a low-risk

326 threshold for boys (>0.45 kg/body mass) and girls ( $>0.36 \mathrm{~kg} /$ body mass) in American

327 adolescents. It is important to note that although the dichotomous (MetS) and continuous method

328 (cardiometabolic risk index) used in this study showed different prevalence, both methods

329 developed identical muscle strength cut points in the diagnosis. Moreover, these age- and sex-

330 specific health-related cut points represented the percentile $25^{\text {th }}$ and the $20^{\text {th }}$ for relative $\mathrm{HG}$ and

331 absolute SLJ. Boys and girls with relative HG or/and absolute SLJ scores below these percentiles

332 had greater odds for MetS and elevated cardiometabolic risk index compared with those reaching

333 the adequate percentiles, independently of cardiorespiratory fitness status. This finding reinforces

334 the idea that an increased cardiometabolic risk is associated with the lowest quartile-quintile of 335 muscle strength in adolescents. ${ }^{30}$ 
337 safe health-related fitness test battery for children and adolescents. ${ }^{7}$ This study included, besides

338 the HG test, the SLJ test to assess skeletal muscle strength. It is also important to highlight that

339 both the HG and SLJ tests are the most used to assess muscle strength in children and

340 adolescents. In fact, these tests are included in a number of field-based fitness test batteries. ${ }^{10}$

341 The observations of the present study are limited by the cross-sectional design nature, and

342 causality cannot be determined. Also, there is a lack of consensus in youth regarding the

343 definition of MetS. We decided to include both dichotomous and continuous methods so that the

344 health-related cut points developed were the most accurate, regardless of the chosen method.

345 Using one method or another could bias the results of the study, given the limitations of each

346 method. However, in the present study, the resulting health-related cut points were the same for

347 each model. Advantages of this study are the proper statistical analysis used (i.e. LMS method

348 and ROC analysis) and the relative large sample of European adolescents, which allow providing

349 age- and sex-specific health-related cut points of upper and lower body muscle strength. It

350 should be noted that although ROC analyses are often used to create diagnostic tests, the first

351 aim of the current study was to identify thresholds that demarcate inadequate/adequate strength

352 relative to cardiometabolic risk factors rather than suggest that strength tests can be used to

353 'diagnose’ MetS.

\section{5. Conclusions}

356 Relative HG and absolute SLJ were the best tests for detecting MetS in European adolescents.

357 Moreover, relative HG appears to be a slightly better test than absolute SLJ to this end. Age- and 358 sex-specific health-related cut points of upper and lower body muscle strength are provided for 
359 European adolescents, which were still predictive of cardiometabolic risk after adjusting for 360 cardiorespiratory fitness. These health-related cut points could be used as a starting point to 361 define adequate levels of upper and lower muscle strength, and the diagnostic statistics provided 362 herein can be used to offer feedback to adolescents, parents, and education and health 363 professionals about what it means to meet or fail the test standards.

364 


\section{Practical implications}

- The present study identifies age- and sex-related health-related upper and lower muscle strength associated with risk of metabolic syndrome in European adolescents.

- Risk of metabolic syndrome is associated with the lowest quartile-quintile of muscle strength in adolescents.

- These health-related cut points might be used as a screening tool to identify adolescents with risk of metabolic syndrome who may benefit from primary and secondary cardiovascular prevention programming. 


\section{REFERENCES}

389 1. Eckel RH, Grundy SM, Zimmet PZ. The metabolic syndrome. Lancet 2005; 365(9468):1415-1428.

391 2. Magnussen CG, Koskinen J, Chen W et al. Pediatric metabolic syndrome predicts 392 adulthood metabolic syndrome, subclinical atherosclerosis, and type 2 diabetes mellitus 393 but is no better than body mass index alone: the Bogalusa Heart Study and the Cardiovascular Risk in Young Finns Study. Circulation 2010; 122(16):1604-1611.

395 3. Ruiz JR, Castro-Pinero J, Artero EG et al. Predictive validity of health-related fitness in 396 youth: a systematic review. Br J Sports Med 2009; 43(12):909-923.

397 4. Smith JJ, Eather N, Morgan PJ et al. The health benefits of muscular fitness for children 398 and adolescents: a systematic review and meta-analysis. Sports Med (Auckland, N.Z.) $3992014 ; 44(9): 1209-1223$.

400 5. Institute of Medicine (IOM). Fitness measures and health outcomes in youth, $401 \quad$ Washington, DC, The national academies press, 2012.

402 6. Moreno LA, De Henauw S, Gonzalez-Gross M et al. Design and implementation of the 403 Healthy Lifestyle in Europe by Nutrition in Adolescence Cross-Sectional Study. Int J $404 \quad$ Obes (Lond) 2008; 32 Suppl 5:S4-11.

$4057 . \quad$ Ruiz JR, Castro-Pinero J, Espana-Romero V et al. Field-based fitness assessment in 406 young people: the ALPHA health-related fitness test battery for children and adolescents. 407 Br J Sports Med 2011; 45(6):518-524.

408 8. Ortega FB, Artero EG, Ruiz JR et al. Physical fitness levels among European 409 adolescents: the HELENA study. Br J Sports Med 2011; 45(1):20-29. 
410 9. Cole TJ, Bellizzi MC, Flegal KM et al. Establishing a standard definition for child

411 overweight and obesity worldwide: international survey. BMJ 2000; 320(7244):1240-

$412 \quad 1243$.

413 10. Castro-Pinero J, Artero EG, Espana-Romero V et al. Criterion-related validity of field-

414 based fitness tests in youth: a systematic review. Br J Sports Med 2009; 44(13):934-943.

415 11. Artero EG, Espana-Romero V, Castro-Pinero J et al. Reliability of field-based fitness $416 \quad$ tests in youth. Int J Sports Med 2011; 32(3):159-169.

417 12. Espana-Romero V, Artero EG, Jimenez-Pavon D et al. Assessing health-related fitness 418 tests in the school setting: reliability, feasibility and safety; the ALPHA Study. Int $J$ $419 \quad$ Sports Med 2010; 31(7):490-497.

420 13. Ruiz JR, Espana-Romero V, Ortega FB et al. Hand span influences optimal grip span in $421 \quad$ male and female teenagers. J Hand Surg 2006; 31(8):1367-1372.

422 14. Leger LA, Mercier D, Gadoury C et al. The multistage 20 metre shuttle run test for 423 aerobic fitness. $J$ Sports Sci 1988; 6(2):93-101.

424 15. Cureton KJ, Plowman SA, Mahar MT. Aerobic capacity assessments, in 425 Fitnessgram/Activitygram Reference Guide. Plowman SA, Meredith MD, ed^eds. 4th ed. 426 Dallas, TX, The Cooper Institute, 2013.

427 16. Nagy E, Vicente-Rodriguez G, Manios Y et al. Harmonization process and reliability 428 assessment of anthropometric measurements in a multicenter study in adolescents. Int $J$

430 17. Gonzalez-Gross M, Breidenassel C, Gomez-Martinez S et al. Sampling and processing of 431 fresh blood samples within a European multicenter nutritional study: evaluation of 
biomarker stability during transport and storage. Int J Obes (Lond) 2008; 32 Suppl 5:S6675.

434 18. Jolliffe CJ, Janssen I. Development of age-specific adolescent metabolic syndrome 435 criteria that are linked to the Adult Treatment Panel III and International Diabetes $436 \quad$ Federation criteria. J Am Coll Cardiol 2007; 49(8):891-898.

437 19. Martinez-Vizcaino V, Ortega FB, Solera-Martinez M et al. Stability of the factorial structure of metabolic syndrome from childhood to adolescence: a 6-year follow-up study. Cardiovasc Diabetol 2011; 10:81.

440 20. Cole TJ, Green PJ. Smoothing reference centile curves: the LMS method and penalized likelihood. Stat Med 1992; 11(10):1305-1319.

442 21. Kelly MJ, Dunstan FD, Lloyd K et al. Evaluating cutpoints for the MHI-5 and MCS using the GHQ-12: a comparison of five different methods. BMC Psychiatry 2008; 8:10.

444 22. Hanley JA, McNeil BJ. A method of comparing the areas under receiver operating 445 characteristic curves derived from the same cases. Radiology 1983; 148(3):839-843.

446 23. Huang TT, Ball GD, Franks PW. Metabolic syndrome in youth: current issues and $447 \quad$ challenges. Appl Physiol Nutr Metab 2007; 32(1):13-22.

448 24. Andersen LB, Lauersen JB, Brond JC et al. A new approach to define and diagnose cardiometabolic disorder in children. J Diabetes Res 2015; 2015:539835.

450 25. Grundy SM, Brewer HB, Jr., Cleeman JI et al. Definition of metabolic syndrome: Report of the National Heart, Lung, and Blood Institute/American Heart Association conference on scientific issues related to definition. Circulation 2004; 109(3):433-438. 
453 26. Calcaterra V, Klersy C, Muratori T et al. Prevalence of metabolic syndrome (MS) in $454 \quad$ children and adolescents with varying degrees of obesity. Clin Endocrinol (Oxf) 2008; $455 \quad 68(6): 868-872$.

456 27. Fernandez Santos JR, Ruiz JR, Gonzalez-Montesinos JL et al. Reliability and Validity of 457 Field-Based Tests to Assess Upper-Body Muscular Strength in Children Aged 6-12 $458 \quad$ Years. Pediatr Exerc Sci 2016; 28(2):331-340.

459 28. Ramirez-Velez R, Pena-Ibagon JC, Martinez-Torres J et al. Handgrip strength cutoff for 460 cardiometabolic risk index among Colombian children and adolescents: The FUPRECOL $461 \quad$ Study. Sci Rep 2017; 7:42622.

462 29. Peterson MD, Zhang P, Saltarelli WA et al. Low Muscle Strength Thresholds for the 463 Detection of Cardiometabolic Risk in Adolescents. Am J Prev Med 2016; 50(5):593-599.

464 30. Artero EG, Ruiz JR, Ortega FB et al. Muscular and cardiorespiratory fitness are 465 independently associated with metabolic risk in adolescents: the HELENA study. Pediatr $466 \quad$ Diabetes 2011; 12(8):704-712. 


\section{Acknowledgements}

We thank the adolescents who participated in the study and their parents and teachers for their collaboration. We also acknowledge the members involved in fieldwork for their efforts and the HELENA study group. The HELENA project was supported by the European Community Sixth RTD Framework Programme (contract FOOD-CT-2005007034). The data for this study was gathered under the aegis of the HELENA project, and further analysis was additionally supported by the Spanish Ministry of Economy and Competitiveness (grants RYC-201416390), the Spanish Ministry of Health: Maternal, Child Health and Development Network (grant RD16/0022), the Fondo Europeo de Desarrollo Regional (MICINN-FEDER) and by the University of Granada, Plan Propio de Investigación 2016, Excellence actions: Units of Excellence; Unit of Excellence on Exercise and Health (UCEES). The funders of the HELENA project had no role in study design, data collection, analysis, or interpretation, writing of the report, or in the decision to submit the paper for publication. The content of this paper reflects the authors' views alone, and the European Community is not liable for any use that may be made of the information contained herein. 
Table 1. ROC-derived cut points and diagnostic statistics for tests of muscle strength to determine elevated cardiometabolic risk index in boys and girls.

\begin{tabular}{|c|c|c|c|c|c|c|c|c|}
\hline Test & $\begin{array}{c}\text { Cut point } \\
\text { Z-score }\end{array}$ & $\begin{array}{l}\text { Cut point } \\
\text { Percentile }\end{array}$ & $\begin{array}{c}\text { Sensitivity } \\
(\%)\end{array}$ & $\begin{array}{c}\text { Specificity } \\
(\%)\end{array}$ & $\begin{array}{l}\text { Youden } \\
\text { Index }\end{array}$ & PPV & NPV & DOR \\
\hline \multicolumn{9}{|l|}{ Boys $(n=444)$} \\
\hline Handgrip (kg) & $>0.143$ & $>55.7$ & 66.7 & 59.9 & 0.27 & 10.8 & 96.1 & 3.0 \\
\hline Rel. Handgrip (kg/mass kg) & $\leq-0.728$ & $\leq 23.3$ & 80.0 & 79.7 & 0.60 & 22.2 & 98.2 & 15.8 \\
\hline Standing Long Jump (cm) & $\leq-0.790$ & $\leq 21.5$ & 73.3 & 80.7 & 0.54 & 21.6 & 97.7 & 11.5 \\
\hline $\begin{array}{l}\text { Rel. Standing Long Jump } \\
\text { (cm x mass kg) }\end{array}$ & $>0.156$ & $>56.2$ & 70.0 & 62.1 & 0.32 & 11.8 & 96.6 & 3.9 \\
\hline \multicolumn{9}{|l|}{ Girls $(n=506)$} \\
\hline Handgrip (kg) & $>-0.287$ & $>38.7$ & 84.2 & 40.8 & 0.25 & 10.4 & 97.0 & 3.6 \\
\hline Rel. Handgrip (kg/mass kg) & $\leq-0.672$ & $\leq 25.1$ & 65.8 & 78.0 & 0.44 & 19.5 & 96.6 & 6.8 \\
\hline Standing Long Jump (cm) & $\leq-0.846$ & $\leq 19.9$ & 44.7 & 83.1 & 0.28 & 17.7 & 94.9 & 4.0 \\
\hline $\begin{array}{l}\text { Rel. Standing Long Jump } \\
\text { (cm x mass kg) }\end{array}$ & $>0.623$ & $>73.5$ & 57.9 & 79.5 & 0.37 & 18.6 & 95.9 & 5.3 \\
\hline \multicolumn{9}{|l|}{ Boys and Girls $(n=950)$} \\
\hline Handgrip (kg) & $>-0.227$ & $>41.0$ & 79.4 & 44.1 & 0.24 & 9.9 & 96.5 & 3.0 \\
\hline Rel. Handgrip (kg/mass kg) & $\leq-0.672$ & $\leq 25.1$ & 72.1 & 78.1 & 0.50 & 20.2 & 97.3 & 9.1 \\
\hline Standing Long Jump (cm) & $\leq-0.790$ & $\leq 21.5$ & 58.8 & 80.5 & 0.39 & 18.9 & 96.2 & 5.9 \\
\hline $\begin{array}{l}\text { Rel. Standing Long Jump } \\
\text { (cm x mass kg) }\end{array}$ & $>0.623$ & $>73.5$ & 54.4 & 79.0 & 0.33 & 16.7 & 95.7 & 4.7 \\
\hline
\end{tabular}

Rel., relative (test expressed by body mass); PPV, positive predictive value; NPV, negative predictive value; DOR, diagnostic odds ratio.

Cardiometabolic Risk Index Status defined by mean of age- and sex-specific z-scores for waist, TG/HDL ratio (triglycerides /highdensity lipoprotein cholesterol), fasting insulin, mean arterial pressure ( $\geq 1.0)$ proposed by Martinez-Vizcaino et al. ${ }^{19}$ 
Table 2. ROC-derived cut points and diagnostic statistics for tests of muscle strength to determine metabolic syndrome in boys and girls.

\begin{tabular}{|c|c|c|c|c|c|c|c|c|}
\hline Test & $\begin{array}{l}\text { Cut point } \\
\text { Z-score }\end{array}$ & $\begin{array}{l}\text { Cut point } \\
\text { Percentile }\end{array}$ & $\begin{array}{l}\text { Sensitivity } \\
(\%)\end{array}$ & $\begin{array}{l}\text { Specificity } \\
(\%)\end{array}$ & $\begin{array}{l}\text { Youden } \\
\text { Index }\end{array}$ & PPV & NPV & DOR \\
\hline \multicolumn{9}{|l|}{ Boys $(n=449)$} \\
\hline Handgrip (kg) & $>-0.129$ & $>44.9$ & 72.7 & 49.1 & 0.22 & 3.5 & 98.6 & 2.6 \\
\hline Rel. Handgrip (kg/mass kg) & $\leq-1.127$ & $\leq 13.0$ & 72.7 & 87.7 & 0.60 & 12.9 & 99.2 & 19.0 \\
\hline Standing Long Jump & $\leq-0.890$ & $\leq 18.7$ & 63.6 & 80.8 & 0.44 & 7.7 & 98.9 & 7.4 \\
\hline $\begin{array}{l}\text { Rel. Standing Long Jump } \\
\text { (cm x mass kg) }\end{array}$ & $>0.156$ & $>56.2$ & 72.7 & 61.2 & 0.34 & 4.5 & 98.9 & 4.2 \\
\hline \multicolumn{9}{|l|}{ Girls $(n=520)$} \\
\hline Handgrip (kg) & -- & -- & -- & -- & -- & -- & -- & -- \\
\hline Rel. Handgrip (kg/mass kg) & $\leq-0.713$ & $\leq 23.8$ & 63.2 & 76.9 & 0.40 & 9.4 & 98.2 & 5.7 \\
\hline Standing Long Jump (cm) & $\leq-0.797$ & $\leq 21.3$ & 57.9 & 78.7 & 0.37 & 9.3 & 98.0 & 5.0 \\
\hline $\begin{array}{l}\text { Rel. Standing Long Jump } \\
\text { (cm x mass kg) }\end{array}$ & $>0.627$ & $>73.5$ & 68.4 & 78.4 & 0.47 & 10.7 & 98.5 & 7.9 \\
\hline \multicolumn{9}{|l|}{ Boys and Girls $(n=969)$} \\
\hline Handgrip (kg) & $>-0.834$ & $>20.2$ & 96.7 & 23.2 & 0.20 & 3.9 & 99.5 & 9.0 \\
\hline Rel. Handgrip (kg/mass kg) & $\leq-0.622$ & $\leq 26.7$ & 70.0 & 73.7 & 0.44 & 7.8 & 98.7 & 6.5 \\
\hline Standing Long Jump (cm) & $\leq-0.797$ & $\leq 21.3$ & 60.0 & 78.6 & 0.39 & 8.2 & 98.4 & 5.5 \\
\hline $\begin{array}{l}\text { Rel. Standing Long Jump } \\
\text { (cm x mass kg) }\end{array}$ & $>0.627$ & $>73.5$ & 56.7 & 77.9 & 0.35 & 7.6 & 98.3 & 4.6 \\
\hline
\end{tabular}

Rel., relative (test expressed by body mass); PPV, positive predictive value; NPV, negative predictive value; DOR, diagnostic odds ratio.

Metabolic Syndrome Status defined by Jolliffe and Janssen. ${ }^{24}$ 
Table 3. Recommended age- and sex-specific cut points to detect elevated cardiometabolic risk index and metabolic syndrome using upper and lower body muscle strength tests.

\begin{tabular}{|c|c|c|c|c|}
\hline \multirow{2}{*}{$\begin{array}{c}\text { Test } \\
\text { Age/Sex }\end{array}$} & \multicolumn{2}{|c|}{$\begin{array}{l}\text { Relative Grip Strength } \\
\qquad(\mathrm{kg} / \mathrm{kg} \text { mass })\end{array}$} & \multicolumn{2}{|c|}{$\begin{array}{l}\text { Standing Long } \\
\text { Jump }(\mathrm{cm})\end{array}$} \\
\hline & Boys & Girls & Boys & Girls \\
\hline 13 years old & 0.44 & 0.41 & 135.4 & 118.1 \\
\hline 14 years old & 0.48 & 0.41 & 151.5 & 121.8 \\
\hline 15 years old & 0.52 & 0.41 & 165.4 & 123.0 \\
\hline 16 years old & 0.56 & 0.42 & 175.9 & 126.0 \\
\hline 17 years old & 0.59 & 0.42 & 184.2 & 129.5 \\
\hline Z-score & \multicolumn{2}{|c|}{$\leq-0.675$} & \multicolumn{2}{|c|}{$\leq-0.842$} \\
\hline Percentile & \multicolumn{2}{|c|}{$\leq 25.0$} & \multicolumn{2}{|c|}{$\leq 20.0$} \\
\hline
\end{tabular}

Youth at or below the values would be considered as having 'poor' muscle strength based on the relevant test. 13 years old $=12.5$ to 13.49 years old, 14 years old $=13.5$ to 14.49 years old, etc. 
Table S1. Descriptive characteristics of the adolescents participating in the study

\begin{tabular}{|c|c|c|c|}
\hline Variable & $\begin{array}{c}\text { Boys } \\
(\mathrm{n}=444)\end{array}$ & $\begin{array}{c}\text { Girls } \\
(\mathrm{n}=506)\end{array}$ & $\begin{array}{c}\text { Total } \\
(\mathrm{n}=950)\end{array}$ \\
\hline Age (years) & $14.7(1.2)$ & $14.7(1.2)$ & $14.7(1.2)$ \\
\hline Stature $(\mathrm{cm})$ & $169.3(9.8)^{*}$ & $161.7(7.1)$ & $165.3(9.3)$ \\
\hline Body Mass (kg) & $61.3(13.7)^{*}$ & $55.6(10.2)$ & $58.3(12.3)$ \\
\hline Overweight/Obese (\%) & $23.9 \% *$ & $18.4 \%$ & $20.9 \%$ \\
\hline Healthy Cardiorespiratory Fitness (\%) & $51.5 \% *$ & $38.9 \%$ & $44.6 \%$ \\
\hline Handgrip Strength $(\mathrm{kg})^{\mathrm{A}}$ & $35.6(9.5)^{*}$ & $25.8(4.9)$ & $30.4(8.9)$ \\
\hline Rel. Handgrip Strength (kg/mass kg) & $0.59(0.12)^{*}$ & $0.47(0.09)$ & $0.53(0.12)$ \\
\hline Standing Long Jump (cm) & $182.8(32.6)^{*}$ & $144.2(25.4)$ & $162.3(34.8)$ \\
\hline Rel. Standing Long Jump (cm x mass $\mathrm{kg}$ ) & $\begin{array}{c}11,226 \\
(3,227)^{*}\end{array}$ & $7,968(1,783)$ & $9,491(3,033)$ \\
\hline Waist Circumference $(\mathrm{cm})$ & $74.2(9.1)^{*}$ & $70.4(7.9)$ & $72.2(8.7)$ \\
\hline Systolic Blood Pressure (mmHg) & $123.9(14.0)^{*}$ & $115.9(11.4)$ & $119.7(13.3)$ \\
\hline Diastolic Blood Pressure (mmHg) & $67.3(8.8)^{*}$ & $68.4(8.7)$ & $67.9(8.8)$ \\
\hline Mean Arterial Pressure (mmHg) & $86.1(9.3)^{*}$ & $84.2(8.7)$ & $85.1(9.0)$ \\
\hline Triglycerides (mg/dL) & $63.1(30.8)^{*}$ & $72.9(37.6)$ & $68.3(34.9)$ \\
\hline HDL-Cholesterol (mg/dL) & $53.1(10.0)^{*}$ & $57.3(10.9)$ & $55.3(10.7)$ \\
\hline TG/HDL Ratio (mg/dL) & $1.28(0.82)$ & $1.36(0.93)$ & $1.32(0.88)$ \\
\hline Fasting Glucose (mg/dL) & $92.9(7.3)^{*}$ & $89.6(6.7)$ & $91.2(7.2)$ \\
\hline Fasting Insulin $(\mu \mathrm{IU} / \mathrm{mL})$ & $10.2(9.2)$ & $10.4(6.8)$ & $10.3(8.0)$ \\
\hline Cardiometabolic Risk Index ${ }^{B}$ & $-0.03(0.67)$ & $-0.01(0.63)$ & $-0.01(0.65)$ \\
\hline Elevated Cardiometabolic Risk Index ${ }^{C}$ & $6.8 \%$ & $7.5 \%$ & $7.2 \%$ \\
\hline Metabolic Syndrome prevalence ${ }^{D}$ & $2.4 \%$ & $3.7 \%$ & $3.1 \%$ \\
\hline
\end{tabular}

Values are Mean (SD), except percentages in the case of Overweight/Obese, Healthy CRF,

cardiorespiratory fitness; and Metabolic Syndrome. Rel., relative; TG/HDL, triglyceride-to-high density 


\section{lipoprotein-cholesterol ratio. \\ * Statistically different from girls $(p<0.05)$.}

A Average of right and left hands. ${ }^{\mathrm{B}}$ Mean of age- and sex-specific z-scores for waist, TG/HDL ratio, fasting insulin, mean arterial pressure. ${ }^{C}$ Cardiometabolic Risk Index $\geq 1.0$, based on Martinez-

Vizcaino. ${ }^{19}$ Detabolic Syndrome based on Jolliffe and Janssen. ${ }^{24}$ 
Table S2. Pairwise comparisons of ROC area under the curve using muscle strength tests to detect elevated cardiometabolic risk index and metabolic syndrome in boys and girls.

\begin{tabular}{|c|c|c|c|c|}
\hline \multirow[b]{2}{*}{ Muscle Strength Parameter } & \multicolumn{2}{|c|}{ Elevated Cardiometabolic Risk Index } & \multicolumn{2}{|c|}{ Metabolic Syndrome } \\
\hline & $\operatorname{AUC}(95 \% \mathrm{CI})$ & $\begin{array}{c}\text { Significantly } \\
\text { different } \\
\text { AUC* }\end{array}$ & $\operatorname{AUC}(95 \% \mathrm{CI})$ & $\begin{array}{c}\text { Significantly } \\
\text { different } \\
\text { AUC* }\end{array}$ \\
\hline Boys & $n=444$ & & $n=449$ & \\
\hline A) Handgrip & $0.655(0.609,0.700)$ & $\mathrm{B}, \mathrm{C}$ & $0.564(0.517,0.610)$ & $\mathrm{B}, \mathrm{C}, \mathrm{D}$ \\
\hline B) Relative Handgrip & $0.832(0.793,0.865)$ & A, D & $0.873(0.838,0.902)$ & A, D \\
\hline C) Standing Long Jump & $0.775(0.733,0.813)$ & A & $0.749(0.706,0.788)$ & $\mathrm{A}$ \\
\hline D) Relative Standing Long Jump & $0.702(0.657,0.744)$ & $\mathrm{B}$ & $0.720(0.675,0.761)$ & A, B \\
\hline Girls & $n=506$ & & $n=520$ & \\
\hline A) Handgrip & $0.640(0.592,0.681)$ & $\mathrm{B}$ & $0.539(0.495,0.582)$ & $\mathrm{B}, \mathrm{D}$ \\
\hline B) Relative Handgrip & $0.748(0.708,0.785)$ & A & $0.755(0.716,0.792)$ & A \\
\hline C) Standing Long Jump & $0.688(0.645,0.728)$ & -- & $0.622(0.620,0.703)$ & -- \\
\hline D) Relative Standing Long Jump & $0.697(0.655,0.737)$ & -- & $0.676(0.634,0.716)$ & A \\
\hline Boys and Girls & $n=950$ & & $n=969$ & \\
\hline A) Handgrip & $0.647(0.615,0.677)$ & $\mathrm{B}, \mathrm{C}$ & $0.549(0.517,0.581)$ & B, C, D \\
\hline B) Relative Handgrip & $0.786(0.758,0.812)$ & $\mathrm{A}$ & $0.799(0.773,0.824)$ & $\mathrm{A}, \mathrm{C}$ \\
\hline C) Standing Long Jump & $0.728(0.698,0.756)$ & $\mathrm{A}$ & $0.695(0.665,0.724)$ & A, B \\
\hline D) Relative Standing Long Jump & $0.699(0.668,0.728)$ & -- & $0.693(0.662,0.721)$ & A \\
\hline
\end{tabular}

$\mathrm{AUC}=$ Area under the receiver operating characteristic (ROC) curve where muscle strength parameter was used to detect presence/absence of metabolic risk/syndrome.

*AUC significantly different from correspondingly labeled test within column based on Hanley and McNeil ${ }^{30}, p<$ 0.05 .

Cardiometabolic Risk Index defined by mean of age- and sex-specific z-scores for waist, TG/HDL ratio, insulin, mean arterial pressure $(\geq 1.0)$ proposed by Martinez-Vizcaino et al. ${ }^{19}$

Metabolic Syndrome defined by Jolliffe and Janssen. ${ }^{24}$ 
Table S3. Logistic regression of elevated cardiometabolic risk index and metabolic syndrome by muscle strength tests.

\begin{tabular}{lcccc}
\hline \multirow{2}{*}{ Model } & \multicolumn{2}{c}{ Relative Grip Strength } & \multicolumn{2}{c}{ Standing Long Jump } \\
\cline { 2 - 5 } & $\begin{array}{c}\text { Cardiometabolic } \\
\text { Risk Index }\end{array}$ & $\begin{array}{c}\text { Metabolic } \\
\text { Syndrome }\end{array}$ & $\begin{array}{c}\text { Cardiometabolic } \\
\text { Risk Index }\end{array}$ & $\begin{array}{c}\text { Metabolic } \\
\text { Syndrome }\end{array}$ \\
\hline $\begin{array}{l}\text { Model 1 } \\
\text { Unadjusted }\end{array}$ & $n=961$ & $n=980$ & $n=954$ & $n=973$ \\
$\begin{array}{l}\text { Model 2 } \\
\text { Adjusted for aerobic fitness } \\
\text { status }\end{array}$ & $7.5(5.0,14.7)$ & $6.2(2.9,13.4)$ & $5.8(3.5,9.6)$ & $4.5(2.3,9.4)$ \\
\hline
\end{tabular}

Values are odds ratio and 95\% confidence interval (OR, 95\% CI), the meeting recommended muscle strength group was referent $(\mathrm{OR}=1.0)$. 